\title{
Solar atmospheric neutrino search with IceCube
}

\author{
The IceCube Collaboration ${ }^{\dagger}$ \\ ${ }^{\dagger}$ http://icecube.wisc.edu/collaboration/authors/icrc17_icecube \\ E-mail: seongjin.in@gmail.com
}

\begin{abstract}
Cosmic rays interacting in the solar atmosphere can produce high energy neutrinos, which could be detected with IceCube. Neutrinos are produced through the decay of pions and kaons, similar to cosmic ray air showers in the Earth's atmosphere. Due to the lower solar atmospheric density, the neutrino spectrum from the Sun is expected to be harder as mesons tend to decay before they can interact or encounter any significant energy loss. The solar disk neutrino flux could be visible over the atmospheric backgrounds at energies above a few hundred GeV. We present IceCube's sensitivity as determined by a dedicated analysis for solar atmospheric neutrinos.
\end{abstract}

Corresponding authors: Seongjin In $^{1 *}$ and Carsten Rott ${ }^{1}$

${ }^{1}$ Department of Physics, Sungkyunkwan University, Seobu 16419, Suwon, South Korea

35th International Cosmic Ray Conference - ICRC2017

10-20 July, 2017

Bexco, Busan, Korea

${ }^{*}$ Speaker. 


\section{Introduction}

Interactions of cosmic rays with the nuclei of the solar atmosphere (photosphere and chromosphere) produce energetic photons. This phenomenon has been described in detail by Seckel et. al. in 1991 [1] and has been recently observed by Fermi-LAT [2]. However, the observed solar disk emission of gamma rays exceeds the theoretical model estimates by an order of magni-

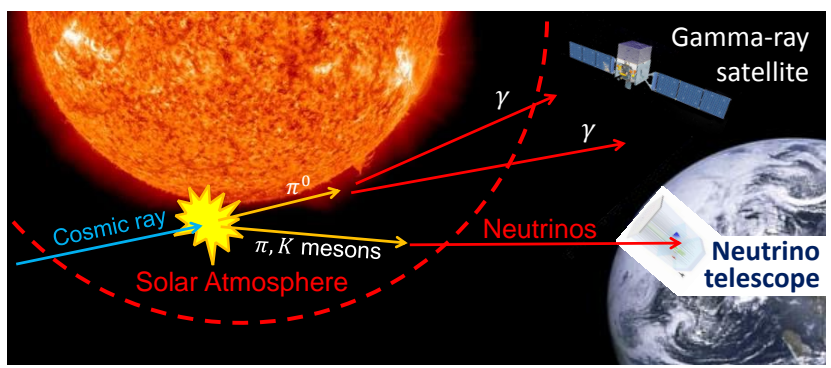

Figure 1: Schematic of solar atmospheric neutrino emission from cosmic ray interactions in the solar atmosphere. tude. The gamma-ray flux anti-correlates with the solar activity and there is evidence that the emission spectrum extends beyond $100 \mathrm{GeV}$ [3].

Cosmic ray interactions in the solar atmosphere are also expected to produce energetic neutrinos (figure 1), which could be detected by terrestrial neutrino telescopes [4]. Solar atmospheric neutrino and gamma-ray observations are important to understand solar magnetic fields and cosmic ray propagation in the inner solar system. Solar atmospheric neutrinos provide a natural background to solar dark matter searches and limit their sensitivity as recently pointed out $[5,6,7]$.

The IceCube observatory, located at the South Pole is the largest neutrino telescope in the world [8]. Construction of the full detector, consisting of 86 strings (IC86), was completed in 2010. The detector is operating very stabile and has opened up a new window to the Universe through the observation of high-energy astrophysical neutrinos. Muon neutrinos above a few hundred $\mathrm{GeV}$ can be reconstructed with sub degree precision. This excellent angular sensitivity gives IceCube also discovery potential for solar atmospheric neutrinos. Previous IceCube analyses have searched for neutrinos in direction of the Sun in an effort to find self-annihilating dark matter [9, 10]. As the Sun is opaque to neutrinos above a few hundred $\mathrm{GeV}$ generated in its center, these analyses were not optimized to look for high energy neutrinos.

In this work, we present the first IceCube analysis that searches for solar atmospheric neutrinos. We first identify an event sample that is well suited for this analysis, the selection is described in section 2. In section 3, we predict the signal and backgrounds based on our simulations. In section 4, we optimize selection criteria and compute sensitivities, before concluding in section 5 .

\section{Identification of optimal event sample}

For our analysis we reuse an existing IceCube event sample. It has the benefit that we can use a sample, which is already well understood and makes our analysis more robust. For the selection of the optimal event sample we do a comparison of signal and background predictions based on effective areas of the corresponding samples.

\subsection{Signal and background flux}

High energy cosmic ray particles entering the solar atmosphere interact with atmospheric nuclei to produce hadronic cascades. When propagating through the atmosphere the cascade products 
decay or interact to produce secondary cascades. Neutrinos can be produced from decays of pions and kaons in the cascades. The processes are similar to the well understood cosmic ray air showers in the Earth atmosphere. However, due to the lower matter density of the solar atmosphere hadrons are more likely to decay than to interact again. This results in a harder neutrino energy spectrum compared to atmospheric neutrinos from the Earth atmosphere.

A theoretical prediction for solar atmospheric neutrinos was given by Ingelman and Thunman (IT) [4], which we adopt as our benchmark signal (see figure 4 of [4]). As background we take the atmospheric neutrino spectrum as determined by IceCube [11]. Since IT only reports the total neutrino flux we apply a factor of $1 / 3$ to correct for neutrino oscillations resulting in equal predictions for $v_{e}$ and $v_{\mu}$. In figure 2 (left) we report signal and background neutrino fluxes as expected from an opening angle around the Sun motivated by the kinematic angle between neutrino and leading lepton and IceCube's angular uncertainty. We use the following energy and flavor dependent angular uncertainties:

$$
A\left(E, v_{i}\right)= \begin{cases}\sqrt{100+900 / E[\mathrm{GeV}]}^{\circ} & v_{e}, \text { for all energies } \\ 30^{\circ} / \sqrt{E / G e V} & v_{\mu}, E<900 \mathrm{GeV} \\ 1^{\circ} & v_{\mu}, E>900 \mathrm{GeV}\end{cases}
$$

Based on the comparison in the figure 2 (left) we expect that the muon neutrino sample will have better sensitivity for our analysis.

\subsection{Event expectation in IceCube}

We estimated the expected number of events with previous IceCube analyses; We compare the effective area of the 3 year solar WIMP analysis [10] and the 7 year likelihood point source analysis [12] in figure 2 (right). For our comparison we only consider up-going events in order to use the Earth as a veto for atmospheric muon backgrounds. The solar WIMP analysis is optimized for low energy neutrinos from the direction of the Sun. Its effective area was not computed for
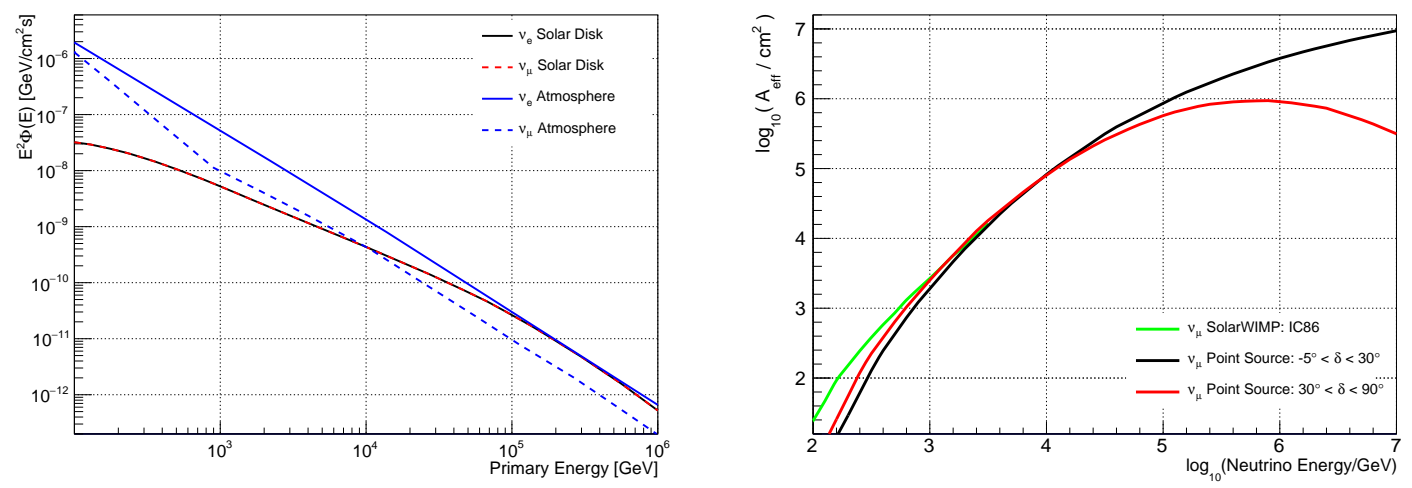

Figure 2: Left: The energy spectrum of atmospheric neutrinos (background) and solar atmospheric neutrinos (signal). An energy dependent search cone with the size of the kinematic angle between lepton and neutrino is applied for backgrounds (blue dotted- $v_{\mu}$ and blue solid- $v_{e}$ ). The spectra of signal (red- $v_{\mu}$ and black- $v_{e}$ ) is identical after the neutrino oscillation applied and apply a $68 \%$ of reconstruction efficiency. Right: Effective areas of solar WIMP analysis line (green) up to $10^{3.5} \mathrm{GeV}$, point source analysis for each declination angle ranges (red and black). 


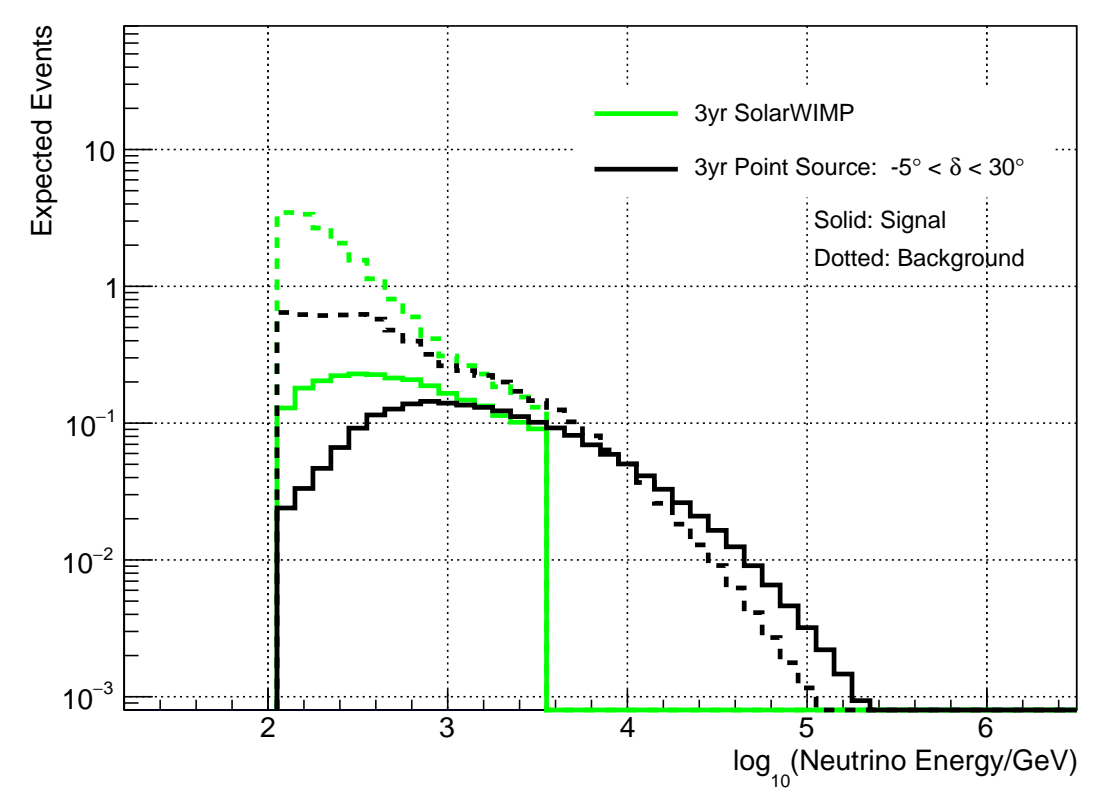

Figure 3: Expected solar atmospheric (solid lines) and atmospheric background (dotted lines) neutrino events as a function of primary neutrino energy. As the solar WIMP analysis focused on low energies where the signal flux is harder, more signal events could be shown, but also more background. With point source sample, the signal and the background are competitive on high energies.

energies above a few $\mathrm{TeV}$ and is expected to fall off around $5 \mathrm{TeV}$ due to a selection cut on the number of hit optical sensor modules. The point source analysis is a full sky analysis that extents to high energies. We use the effective area for the $-5^{\circ}<\delta<30^{\circ}$ declination cut.

We estimate the number of signal and background events as function of true muon neutrino energy using the effective areas and the neutrino flux from figure 2. We assumed that the signal is uniformly distributed within the radius of the Sun $\left(0.25^{\circ}\right)$ and $68 \%$ of the signal can be reconstructed in the direction of the Sun within the cone opening angle.

Figure 3 shows the energy distribution of the expected events with 3 years of IceCube data. The actual livetimes of the samples are 528.3 and 630 days, for the solar WIMPs (green) and point source sample (black), respectively. The solar WIMP analysis yields a higher signal acceptance than the point source analysis however most events are expected in the low energy region $\sim$ $\mathrm{O}(100 \mathrm{GeV})$. Overall the signal to background ratio for the solar WIMP analysis $(S / B=0.147)$ is less favorable compared to the point source analysis $(S / B=0.309)$. We decide to use the point source sample for our analysis as it is expected to yield the best sensitivity.

\section{Monte Carlo Simulation}

According to the estimate in section 2 we now use the point source Monte Carlo (MC) simulation samples for our analysis. For the signal simulation, we re-weighted the simulation to the theoretical model of the signal in [4]. In this section, we describe the signal and background simulation results. 


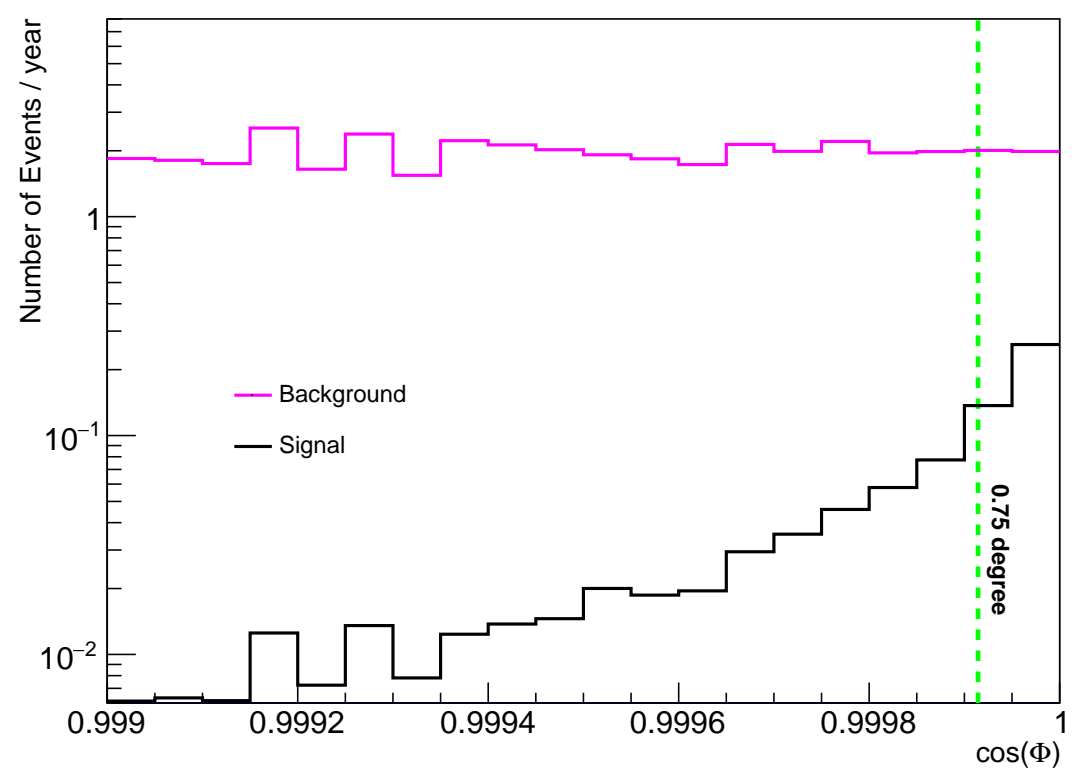

Figure 4: The expected number of events as a function of the reconstructed angular distance from the Sun. The Sun is located at $\cos (\Phi)=1$. The magenta line is the simulated background and black is the re-weighted signal.

\subsection{Atmospheric Background Neutrinos}

We simulated $v_{\mu}$ in the IC86 configuration from $100 \mathrm{GeV}$ to $1 \mathrm{EeV}$ for the whole sky. Our theoretical model for atmospheric neutrinos is the Honda 2006 flux from [13]. As mentioned in section 2, the Earth can be used as a $\mu$ veto and we only used the up-going samples. In our simulation we predict $66319.4 \pm 0.6$ atmospheric $v_{\mu}$ up-going events per year.

\subsection{Solar Atmospheric Neutrinos}

The IT spectrum is our benchmark signal and we used the parameterization in [4]. Table 2 and eq 3.1 are taken from table 2 and eq.15 in [4] for the $v_{\mu}$ flux.

$$
\Phi(E)= \begin{cases}N_{0} E^{-\gamma-1} /(1+A E) & E<E_{0} \\ N_{0}^{\prime} E^{-\gamma^{\prime}-1} /(1+A E) & E>E_{0}\end{cases}
$$

For the signal samples, the simulations are re-weighted into eq 3.1. Since the declination of the Sun is from $23.44^{\circ}$ to $-23.44^{\circ}$ at South Pole, the zenith angle $(\Theta)$ of the Sun is fixed according to eq 3.2.

$$
\Theta=\left(90^{\circ}-23.44^{\circ} * \cos (360 / 365 * \text { day })\right)
$$

The azimuth angle is randomly scrambled. Only events which are within the solar angular extension of $0.25^{\circ}$ are selected. We expect $2.85 \pm 0.01$ signal events in 3 years of data. Note that we only consider upgoing events, which limits our selection to 1.5 years, when the Sun is below 


\begin{tabular}{ccccccc}
\hline & $N_{0}$ & $\gamma$ & $\mathrm{A}$ & $E_{0}$ & $\gamma$ & $N_{0}{ }^{\prime}$ \\
\hline$v_{\mu}+\bar{v}_{\mu}$ & $1.3 \times 10^{-5}$ & 1.98 & $8.5 \times 10^{-6}$ & $3.0 \times 10^{6}$ & 2.38 & $5.1 \times 10^{-3}$ \\
\hline$v_{e}+\bar{v}_{e}$ & $7.4 \times 10^{-6}$ & 2.03 & $8.5 \times 10^{-6}$ & $1.2 \times 10^{6}$ & 2.33 & $5.0 \times 10^{-4}$ \\
\hline
\end{tabular}

Table 1: Fitting Parameters for the Ingelman \& Thunman spectrum [4] and used in this analysis.

the horizon. Figure 4 shows our background and signal distribution along the reconstructed angular distance with respect to the position of the Sun. As the signal events are spread within $0.25^{\circ}$, the signal events are concentrated in the last bin (see figure 4).

\section{Sensitivity Estimate}

\subsection{Selection Cut Optimization}

We conduct an optimization of the opening angle and energy cuts to improve our sensitivity. As a first effort, we calculated $\mathrm{S} / \sqrt{B}$ as a function of the cone opening angle and the reconstructed energy cut, in figure 5. The highest (black star) $\mathrm{S} / \sqrt{B}$ is found with an opening angle cut of $1.5^{\circ}$ and an energy cut of $430.0 \mathrm{GeV}$. The z-axis of figure 5 is flat below a few hundreds of $\mathrm{GeV}$, because the reconstructed energy dominantly has spread at $\mathrm{TeV}$ region. For the best sensitivity, we apply these cuts to our samples. Note that we did not simulate neutrino events with energies below 100 $\mathrm{GeV}$. Sometimes these events could reconstruct to higher energies and skew our optimization. We

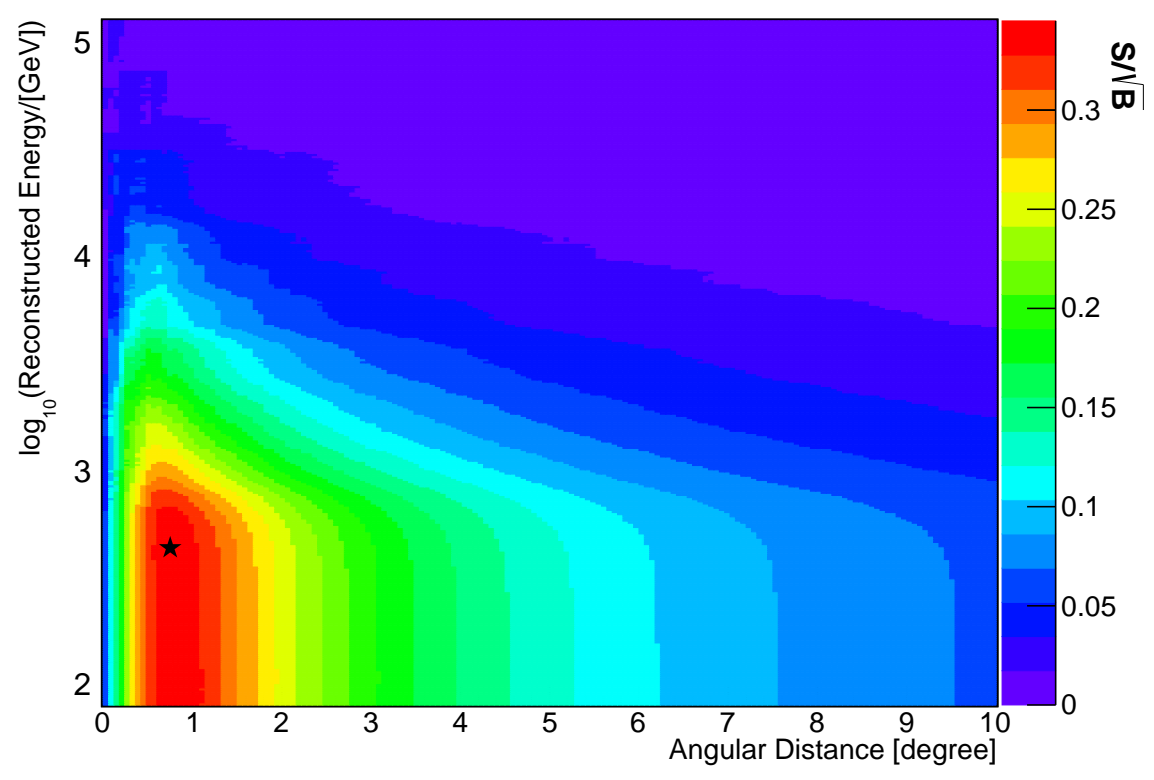

Figure 5: Signal(S) and background(B) ratio plot for the corresponding angular distance from the Sun and energy cut. The color bar represents $\mathrm{S} / \sqrt{B}$. The black star indicates the optimal selection cut, which yields the highest $S / \sqrt{B}$ of 0.34 . 

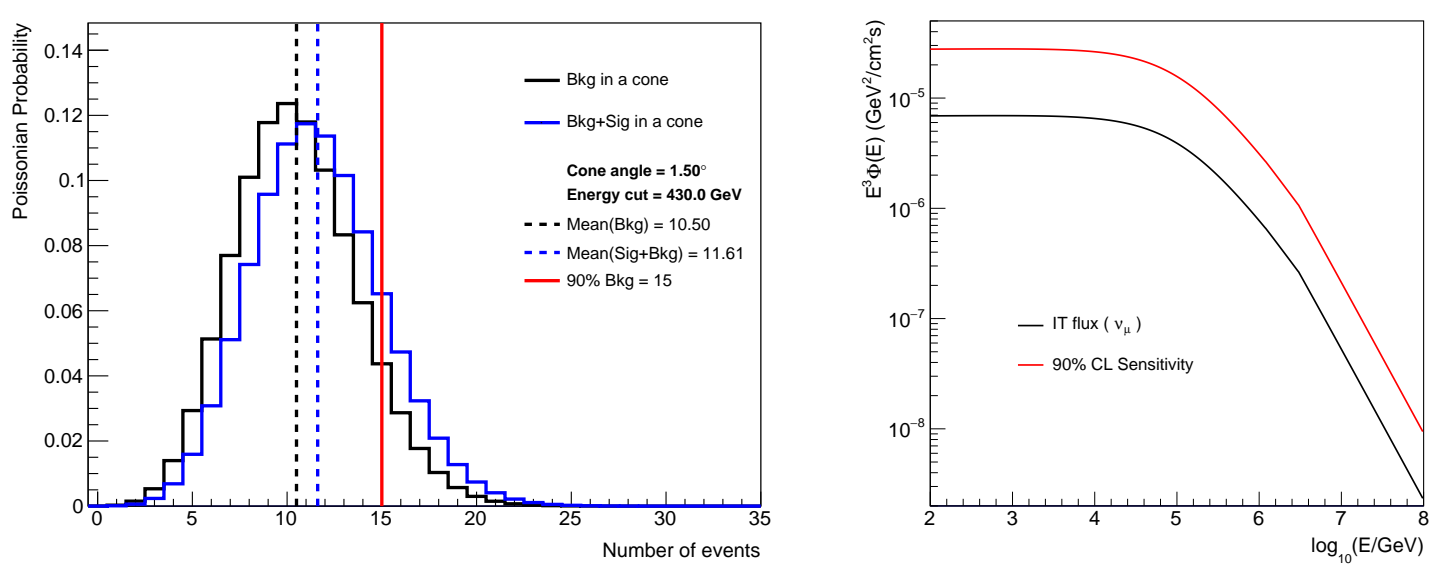

Figure 6: Left: Poisson distribution for the expected number of background events (black), signal+background (blue) within a $1.5^{\circ}$ opening angle cone. The vertical black dotted and blue dotted lines are the median of each distribution. The vertical red line denotes the $90 \%$ lower quantile of the background only distribution. Right: Red line is the $90 \%$ confidence level sensitivity. The black line is the IT spectrum scaled down by a factor of 3 by the effect of neutrino oscillation.

estimate that the contribution of these events is less than $4 \%$ of our event sample above the optimal selection cut.

\subsection{Sensitivity}

The estimated number of background events can be utilized to calculate a sensitivity to the solar atmospheric neutrino flux. We calculate a sensitivity at $90 \%$ confidence level using a Poisson distribution. According to the cuts in section 4, simulated events are selected with reconstructed energies above $430 \mathrm{GeV}$ and reconstructed direction within $1.5^{\circ}$ from the Sun. With these cuts, $10.5 \pm 0.18$ background events $\left(n_{b k g}\right)$ and $1.12 \pm 0.19$ signal events $\left(n_{s i g}\right)$ are expected in 3 years from the direction of the Sun.

The left hand side of figure 6 shows a probability density function for pure background, background and the expected signal $\left(n_{b k g+s i g}=12.11\right)$ and the medians of these distributions. The lower $90 \%$ quantile of the pure background distribution is found at $n_{90}=15$. The right-hand side of figure 6 is the statistical sensitivity of IceCube from the point source up-going simulation sample. The black line represents the IT spectrum taking into account neutrino oscillations. Given that the $90 \%$ confidence level sensitivity exceeds the expected signal no observation is expected with the current analysis. However with an improved event selection the signal might be in reach. For simplicity we used a cut and count analysis, for the actual analysis we are developing a log-likelihood method similar to the approach used in the solar dark matter analysis [10].

\section{Summary}

We looked at the sensitivity of IceCube to solar atmospheric neutrinos. We have estimated the expected number of signal and background events from the direction of the Sun with the effective areas of previous IceCube analyses in section 2. We determined that the point source analysis 
is better suited compared to the solar WIMP analysis. We calculated the signal and background expectations using the point source analysis [12] MC simulation sample.

With up-going MC simulation samples of the point source analysis, we estimated the number of signal and background events using the optimized cuts. For the optimization of the cuts, we used $\mathrm{S} / \sqrt{B}$ to determine the cone cuts and the energy cuts. The maximum value of $\mathrm{S} / \sqrt{B}$ is 0.34 at a $1.5^{\circ}$ opening angle cut and a $430 \mathrm{GeV}$ energy cut. This yields $n_{s i g}=1.12$ and $n_{b k g}=10.5$ events in 3 years. The IceCube sensitivity for the solar atmospheric neutrino flux is obtained (figure 6). The sensitivity is expected to further improve in the future by using a log-likelihood analysis method, expanding the analysis to include additional neutrino flavors, and by utilizing down-going events. Further selection cut optimization could also help to distinguish the signal better from backgrounds.

It should be noted that there is a considerable uncertainty on the neutrino flux prediction from the Sun $[4,7,5]$, making the outcome of this search less predictable. The analysis carries importance to help understand solar magnetic fields and cosmic ray propagation in the inner solar system. An observation of the solar atmospheric neutrino could be the first high energy astrophysical point source. Solar atmospheric neutrinos also pose a background for solar Dark Matter searches [7, 5, 6] and this analysis can help quantify it.

\section{References}

[1] Seckel. D, Stanev T. and Gaisser. T. K., Astrophys J. 382 (1991) 652-666.

[2] Fermi-LAT Collaboration, A.A. Abdo et al., Astrophys J. 734 (2011) 116.

[3] Kenny C.Y. Ng, John F. Beacom, Annika H.G. Peter and C. Rott, Phys. Rev. D94 (2016) 023004.

[4] G. Ingelman and M. Thunman, Phys. Rev. D54 (1996) 4385-4392.

[5] C.A. Argüelles, G. de Wasseige, A. Fedynitch and B.J.P. Jones, [astro-ph/1703.07798].

[6] Kenny C. Y. Ng, John F. Beacom, Annika H. G. Peter and C. Rott, [astro-ph/1703.10280].

[7] J.Edsjö, J.Elevant, R.Enberg and C.Niblaeus, [astro-ph/1704.02892v1].

[8] IceCube Collaboration, M.G. Aartsen et al., JINST 12 (2017), P03012.

[9] IceCube Collaboration, M.G. Aartsen et al., Phys. Rev. Lett. 110 (2013) 131302.

[10] IceCube Collaboration, M.G. Aartsen et al., Eur. Phys. J. C77 (2017) 146.

[11] IceCube Collaboration, M.G. Aartsen et al., Phys. Rev. D91 (2015) 122004.

[12] IceCube Collaboration, M.G. Aartsen et al., Astrophys J. 835 (2017) 151.

[13] M. Honda, T.Kajita, K.Kasahara, S.Midorikawa and T. Sanuki, Phys. Rev. D75 (2007) 043006. 